\section{Arsenic speciation in fish products and seafood as a prerequisite for proper risk assessment}

\author{
Pierluigi Piras, ${ }^{1}$ Roberta Orletti,, \\ Giannina Chessa, ${ }^{3}$ Cristiano Carloni, ${ }^{2}$ \\ Francesco Griffoni, ${ }^{2}$ Paolo Palombo, ${ }^{2}$ \\ Francesco Velieri ${ }^{2}$
}

'Servizio Veterinario di Igiene degli Alimenti di Origine Animale, Azienda Sanitaria Locale n. 7, Regione Autonoma della Sardegna, Carbonia (CI); IIstituto Zooprofilattico Sperimentale Umbria e Marche, Ancona; ${ }^{3}$ Istituto Zooprofilattico Sperimentale della Sardegna G. Pegreffi, Sassari, Italy

\section{Abstract}

The Boi Cerbus lagoon, facing a mining and industrial site in Sardinia (Italy), is an important fishing area for the local population. Previous studies showed high concentrations of total arsenic $\left(\mathrm{As}_{\mathrm{tot}}\right)$ in fish, molluscs and crustaceans sampled in the lagoon, and a possible exceeding of the provisional tolerable weekly intake set by the Joint FAO/WHO Expert Committee on Food Additives by some local consumer groups. However, the percentage of inorganic $\mathrm{As}\left(\mathrm{As}_{\text {inorg }}\right)$ should be known for a correct assessment of potential risk, as its toxicity is much higher than that of the organic forms Eighty samples of 14 different species of fish, molluscs and crustaceans, sampled in the Boi Cerbus lagoon in 3 different seasons (winter, spring and summer), were analysed for $\mathrm{As}_{\text {tot }}$ by inductively coupled plasma mass spectrometry (ICP-MS) and $\mathrm{As}_{\text {inorg }}$ by high performance liquid chromatography-ICP-MS. All the data obtained from the analysis were statistically processed to evaluate significant differences based on season, taxon and habitat, in preparation for a subsequent risk assessment.

\section{Introduzione}

L'arsenico (As) è un metalloide associato a diverse rocce, specialmente a minerali contenenti solfuro, rivelandosi molto mobile nell'ambiente: l'erosione delle rocce converte infatti i solfuri di As in ossido arsenioso che entra nel ciclo ambientale contaminando i corpi idrici e, attraverso la catena alimentare, distribuendosi diffusamente in tutto il regno vegetale e animale. Si tratta quindi di un elemento ubiquitario, già naturalmente presente nei suoli, nelle acque, nei sedimenti e negli organismi. Dall'avvento della metallurgia, ai livelli di fondo dell'As ambientale si sono aggiunti quelli antropogenici, in quanto esso rappresenta anche un sottoprodotto nell'estrazione dei metalli, mentre il contributo antropogenico recente alle concentrazioni di As ambientale è derivato soprattutto dall'uso di pesticidi a base di suoi composti. Va perciò sottolineato come la concentrazione di As nella crosta terrestre presenti notevoli variazioni geografiche, anche dipendenti dal tipo di rocce, ed è nota l'importanza di conoscere i processi che controllano la mobilità e la speciazione dell'As nei suoli, nelle acque e nel biota. Spesso, infatti, la forma o specie chimica di un determinato elemento chimico è importante quanto la sua quantità e ciò vale in particolar modo per l'As, in quanto la pericolosità per l'uomo delle sue diverse forme varia in modo davvero considerevole (Sharma e Sohn, 2009). La valenza sanitaria del problema è ancora più evidente dal quadro che ci viene fornito dalla dinamica delle diverse forme chimiche dell'As in ambiente acquatico marino. L'As disciolto in acqua di mare si trova infatti prevalentemente in forma di arsenato $[\mathrm{As}(\mathrm{V})]$, mentre l'arsenito [As(III)], potenzialmente più tossico e cancerogeno, rappresenta raramente più del $20 \%$ (Neff, 1997) dell'arsenico totale $\left(\mathrm{As}_{\mathrm{tot}}\right)$. Oltre alle forme inorganiche, l'As in ambiente marino è presente in varie forme organiche, a partire da quelle mono-, di- e tri-metilate (Figura 1). Le alghe marine assorbono facilmente l'arsenato dall'acqua ed in esse si riscontra la presenza di diversi composti organici arseno-ribosidici. Tali composti, spesso indicati col termine generico di arsenozuccheri (Figura 1), rappresentano un anello importante nel ciclo biochimico dell'As in ambiente marino (Kirby et al., 2005). Costituiscono infatti la forma prevalente di As nelle alghe (circa $3 / 4$ della frazione di As), ma sono presenti in quantità significative anche nei molluschi marini, in particolare nei gasteropodi erbivori (circa $1 / 4$ della frazione di $\mathrm{As}_{\text {tot }}$ ) e, viceversa, sono presenti in bassissime concentrazioni nei pesci. Gli studi sulla tossicità degli arsenozuccheri risultano quindi numerosi e di grande interesse anche in ambito europeo, per il crescente uso alimentare delle alghe, consolidato invece per tradizione nei paesi asiatici. Tuttavia, nonostante la notevole mole di dati bibliografici, non ci sono ancora informazioni definitive sulla eventuale tossicità di questi composti (Francesconi, 2010). Gli animali acquatici marini (pesci, crostacei e molluschi acquatici) hanno invece una capacità limitata di bioconcentrare arsenico inorganico $\left(\mathrm{As}_{\text {inorg }}\right)$ dall'acqua marina, mentre possono bioaccumulare e biotrasformare i composti organici dell'As attraverso la catena alimentare. Tutti i tessuti, sia degli invertebrati che dei pesci marini, contengono infatti
Correspondence: Pierluigi Piras, Servizio Veterinario di Igiene degli Alimenti di Origine Animale, Azienda Sanitaria Locale n. 7, Regione Autonoma della Sardegna, Piazza Cagliari s.n.c., 09013 Carbonia (CI), Italy.

Tel. +39.339.2056525 - Fax: +39.0781.660614.

E-mail: pirasp@tiscali.it

Key words: Arsenic, Elements speciation, Fishing products, Food safety.

Acknowledgments: the Authors thank Dr. Antonino Bella, researcher at the Istituto Superiore di Sanità of Rome, whose assistance in statistical analysis has been extremely helpful. Funding: this paper has been funded by the Italian Board of Health (project no. IZSUM RC005 2011).

Received for publication: 16 July 2014.

Revision received: 12 October 2014.

Accepted for publication: 16 October 2014.

This work is licensed under a Creative Commons Attribution 3.0 License (by-nc 3.0).

CC Copyright P. Piras et al., 2015

Licensee PAGEPress, Italy

Italian Journal of Food Safety 2015; $4: 4577$

doi:10.4081/ijfs.2015.4577

concentrazioni molto elevate di As (Neff, 1997), ma questo si trova per lo più nella forma organica. Gli organismi marini sembra infatti che abbiano affrontato evolutivamente il problema sfruttando la ricca chimica organica dell'As, per trasformare le forme inorganiche in una gamma di composti organici, fondamentalmente atossici (Francesconi e Edmonds, 1998). Il composto più abbondante è l'arsenobetaina, con quantità residuali di arsenocolina (Figura 1). In tutta la copiosa bibliografia scientifica disponibile (Francesconi, 2010), l'arsenobetaina e l'arsenocolina sono considerate non tossiche, sebbene la prima sia stata molto più studiata e ne sia stata verificata l'inerzia metabolica, mentre sull'arsenocolina (che, comunque costituisce generalmente meno dell' $1 \%$ dell'As $s_{\text {tot }}$ presente in organismi marini) esistono ancora lacune conoscitive. Appare chiaro perciò come le determinazioni delle sole concentrazioni dell'As $s_{\text {tot }}$ nelle diverse matrici ambientali (sedimento, acqua e soprattutto biota) non possano, da un lato, costituire un elemento sufficiente per poter valutare il possibile effetto sugli organismi e la dinamica di tale metalloide nell'ambiente marino-costiero considerato (Sörös et al., 2003; Fattorini et al., 2004; Foster et al., 2005; Fattorini et al., 2006) e, dall'altro, non essere sufficienti per lo svolgimento di una corretta valutazione del rischio umano per esposizione alimentare a prodotti della pesca contaminati da tale metalloide (De Gieter et al., 2002; 
Greene e Crecelius, 2006). Benché le forme inorganiche dell'As siano più tossiche di quelle organiche, la quasi totalità dei dati sulla presenza di As negli alimenti, raccolti nel quadro dei controlli ufficiali in ambito europeo, ripor-

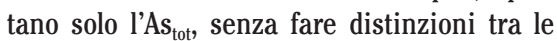
sue varie forme. Poiché, proprio nei prodotti della pesca l'As è prevalentemente presente in forme organiche, è stata evidenziata la necessità di disporre di dati di speciazione (EFSA, $2009,2014)$, anche in considerazione del fatto che un risk assessment che non tenesse conto di ciò, condurrebbe a sovrastimare notevolmente il rischio sanitario. Gli organismi marini presentano infatti concentrazioni notoriamente molto alte di $\mathrm{As}_{\text {tot }}$, ma con una prevalenza di composti innocui (come l'arsenobetaina in pesci, crostacei e molluschi), o ritenuti tali (quali gli arsenozuccheri in molluschi ed alghe), bioaccumulati secondo un complesso ciclo bio-geochimico (Figura 2). I composti dell'As presenti negli ambienti acquatici marini sono quindi diverse decine ed è nota la variabilità della capacità dei diversi organismi vegetali e animali, anche in relazione al livello trofico da questi occupato (Kirby e Maher, 2002), di bioaccumulare alcuni di questi composti in luogo di altri (Rahman et al., 2012). In particolare quindi, è l'analisi selettiva delle forme inorganiche da quelle organiche dell'As a rivestire un'importanza cruciale nella valutazione del rischio legato all'esposizione a tale elemento attraverso la dieta, in quanto tali diverse forme chimiche presentano notevolissime differenze nella tossicità (Neff, 1997; Francesconi, 2010). I composti inorganici dell'As sono infatti potenti cancerogeni per l'uomo e, inoltre, è ampiamente dimostrato che l'esposizione cronica ad essi determina un aumento nel rischio d'insorgenza di numerose altre patologie non tumorali, come ipercheratosi, ipertensione, diabete, nonché disturbi respiratori, neurologici e cardiovascolari (Saha et al., 1999). È noto inoltre che la caratteristica più importante che determina la tossicità dell'As $s_{\text {inorg }}$ è il suo stato di ossidazione: dati di letteratura indicano infatti che l'As sotto forma di arsenito [As(III)] è da 10 a 60 volte più tossico della forma di arsenato $[\mathrm{As}(\mathrm{V})]$ e da 70 a 100 volte più tossico delle specie organiche metilate (Borak e Hosgood, 2007). Quali forme chimiche siano implicate, se organiche 0 inorganiche, rappresenta quindi il fattore determinante per quanto riguarda la valutazione della tossicità, oltre che la biodisponibilità, dell'As (Moreda-Piñeiro et al., 2012). Ad oggi sarebbero più di cinquanta le forme di As organico identificate in organismi marini (Francesconi, 2010), nei tessuti dei quali, oltre a tutta la gamma fin'ora descritta, si aggiungono pure i composti lipidici (Sele $e t$ $a l ., 2012)$. Negli oli di pesce è stata infatti da tempo rilevata una serie di acidi grassi saturi con la porzione terminale dimetil-arsinoil-
(Figura 1). La presenza di arsenolipidi in oli di pesce non sembra tuttavia sollevare problemi di tossicità nell'uomo: benché essi vengano assorbiti dal tratto gastrointestinale, l'As è infatti rapidamente escreto sotto forma di metaboliti organici idrosolubili (prevalente- mente composti dimetilati ed arsenobetaina). In sintesi, per quanto riguarda le forme organiche dell'As, è sufficientemente assodato come l'arsenobetaina, che è la forma predominante nei prodotti della pesca, non desti alcuna preoccupazione dal punto di vista tossicolo-

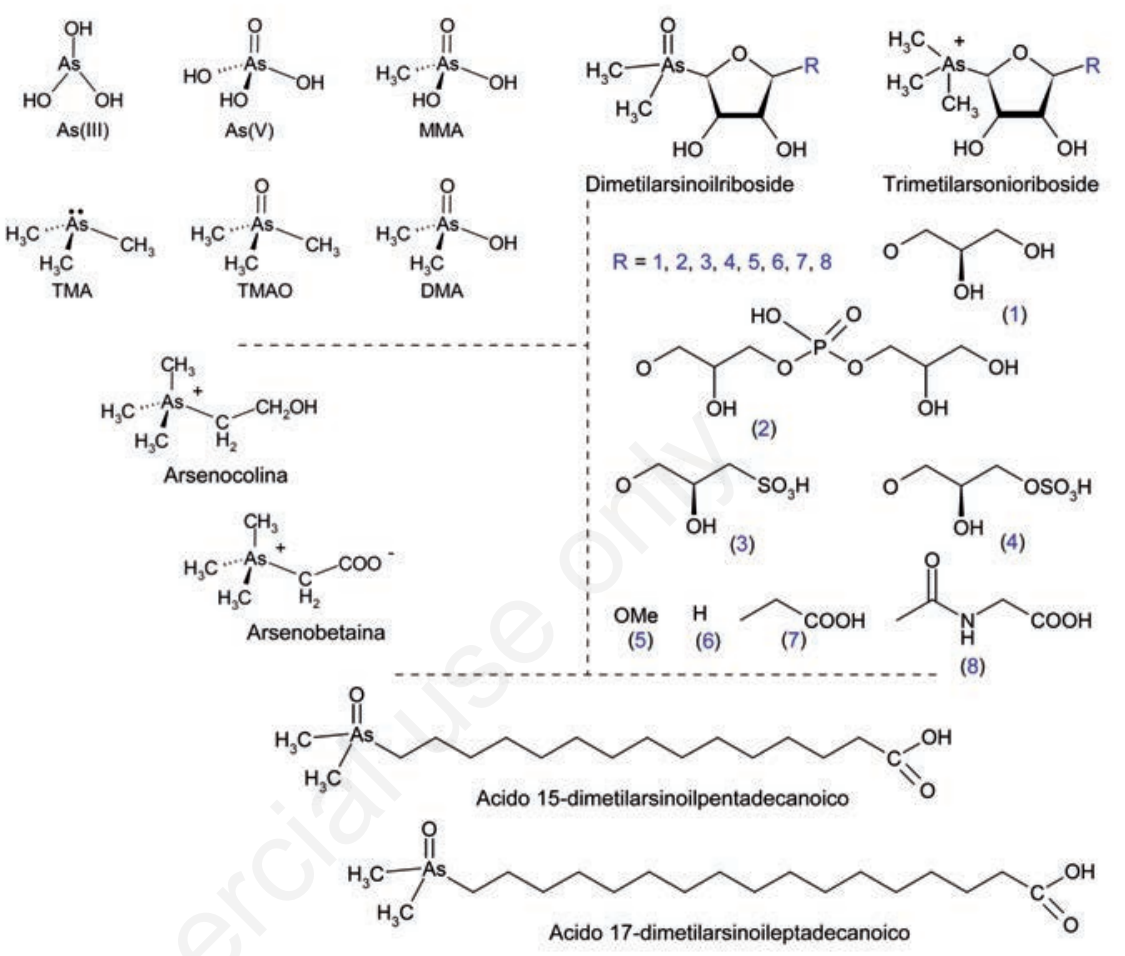

Figura 1. Rappresentazioni strutturali dell'acido arsenioso [As(III)], dell'acido arsenico [As(V)], dell'acido monometilarsonico (MMA), dell'acido dimetilarsinico (DMA), della trimetilarsina (TMA) e del suo ossido (TMAO), degli arsenoribosidi metilati e delle loro $R$ varianti, dell'arsenocolina e dell'arsenobetaina ed esempi di arsenolipidi (C-15 e C17).

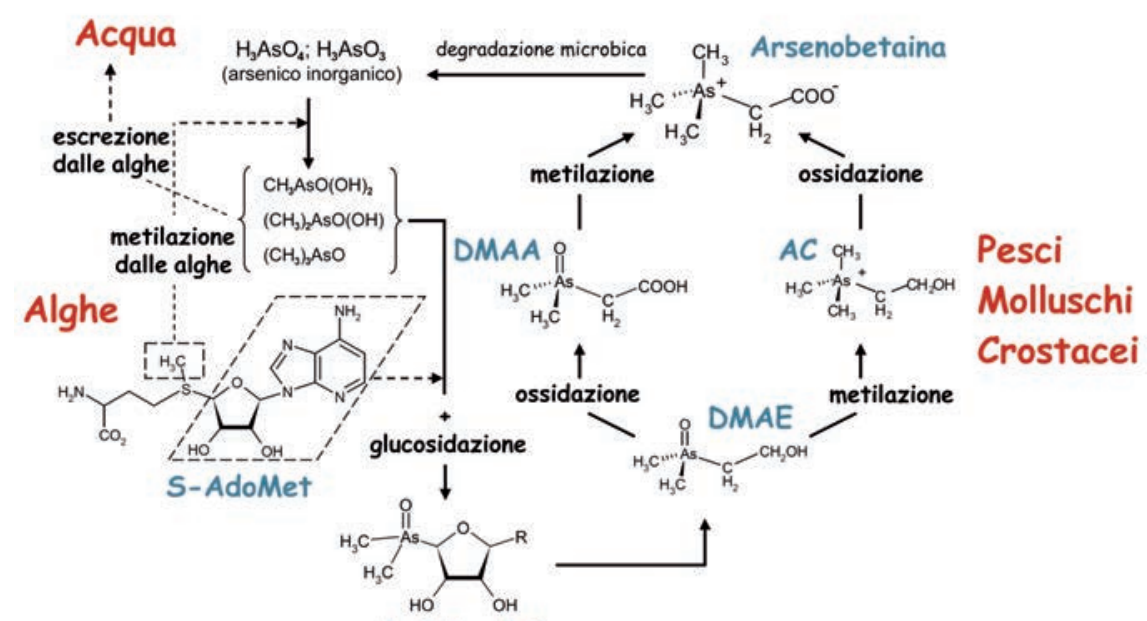

arsenozuccheri dimetilati

Figura 2. Ciclo dell'arsenico in ambiente marino (modificato da Edmonds e Francesconi, 1987). 
gico e che, relativamente agli arsenozuccheri e agli arsenolipidi (poiché vengono nell'uomo generalmente metabolizzati in dimetilarsinato), non siano attualmente disponibili sufficienti informazioni sull'eventualità e grado di una qualche loro tossicità residuale.

In un precedente studio (Piras e Mulas, 2011) sul contenuto di metalli pesanti e metalloidi in molluschi bivalvi, crostacei e pesci lagunari campionati nell'area d'indagine, la laguna di Boi Cerbus nella parte sud-occidentale della Sardegna (Sulcis-Iglesiente), erano stati rilevati elevati livelli di As ed erano state avanzate stime per la valutazione del rischio da esposizione alimentare. Seguendo l'approccio metodologico armonizzato europeo, che utilizza il parametro tossicologico del provisional tolerable weekly intake fissato dal Joint FAO/WHO Expert Committee on Food Additives, le concentrazioni di $\mathrm{As}_{\text {tot }}$ riscontrate avrebbero contribuito di 2,15 volte la dose accettabile nella classe d'età dei bambini e di 1,26 volte in quella dei giovani. Si è trattato ovviamente di stime molto prudenziali, nell'ipotesi estrema che tutto l'As rilevato avesse

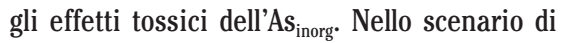
ricerca che si è aperto, è apparso quindi necessario poter disporre di tecniche analitiche capaci di discriminare, in una consistente gamma di prodotti della pesca, l'As inorg $_{\text {dal }}$ complesso di composti riferibili alle forme organiche. I metodi disponibili per l'analisi di speciazione si basano fondamentalmente sulla combinazione di tecniche di separazione con quelle di rivelazione. Nel caso delle analisi di speciazione dell'As (Le et al., 1994; Goessler et al., 1997; De Gieter et al., 2002; Gong et al., 2002; Sörös et al., 2003; Francesconi e Kuehnelt, 2004) le tecniche di separazione attualmente più diffuse sono quelle cromatografiche HPLC in combinazione (o hyphenated applications) con rivelatori di massa ICP. L'analisi di speciazione dell'As in campioni marini è stata però generalmente eseguita per ricerche ad hoc $\mathrm{e}$ finalità scientifiche (comunque non nel contesto di piani di monitoraggio/sorveglianza), ponendosi come obiettivo soprattutto la differenziazione multipla delle specie organiche dell'elemento (Hirata et al., 2006; MoredaPiñeiro et al., 2012). Con un approccio che meglio si presta ad obiettivi ed operatività della sanità pubblica, nel presente studio si è invece adottata una particolare procedura di preparazione del campione che permette, sempre attraverso tecnica cromatografia liquida ad alta prestazione-spettrometria di massa a plasma accoppiato induttivamente (high performance liquid chromatography-inductively coupled plasma mass spectrometry; HPLC-ICPMS), un'analisi di speciazione semplificata, ovvero finalizzata alla sola determinazione dell'As $_{\text {inorg. }}$ Originariamente sperimentata in campioni di pesci marini (Larsen et al., 2005), dopo un'ulteriore semplificazione e perfezionamento, è stata applicata, anche sui molluschi bivalvi e crostacei (Sloth et al., 2005) ed è stata recentemente implementata presso il laboratorio Controllo Chimico e Biomonitoraggio della Sezione di Ancona dell'IZS-UM, dove sono state svolte le analisi di speciazione del presente studio. Nell'ambito delle attività di biomonitoraggio ambientale effettuate nella laguna di Boi Cerbus, si è quindi voluta sviluppare la ricerca nell'area costiera indagata, con lo scopo di rilevare le frazioni tossiche dell'As (come $\mathrm{As}_{\text {inorg }} / \mathrm{As}_{\text {tot }} \mathrm{x}$ 100) su organismi marini sentinella, rappresentati da una diversificata gamma di specie marino-lagunari edibili, ponendo particolare attenzione ad evidenziare eventuali differenze interspecifiche 0 tra taxa, oltre che tra organismi bentonici (o a contatto col sedimento) vs quelli nectonici, ed approfondire le conoscenze sul comportamento dell'As in organismi marino-costieri con habitat prossimo ad aree minerarie, come quelle del Sulcis-Iglesiente, e valutarne l'impatto sulla sicurezza alimentare.

\section{Materiali e Metodi}

Utilizzando la sopra riferita procedura di speciazione basata su tecnica accoppiata HPLC/ICP-MS (Sloth et al., 2005), si è studiata la distribuzione dell'As inorg $_{\text {(e sua quantifica- }}$ zione proporzionale sull' $A s_{\text {tot }}$ ) in $n^{\circ} 80$ campioni, riferibili a ${ }^{\circ} 14$ specie acquatiche marine eduli (Tabella 1) della laguna di Boi Cerbus pescate/raccolte nel corso di tre calate del: 21 febbraio, 29 aprile e 6 luglio del 2013. Gli organismi acquatici sono stati campionati nella sola parte edibile ed immediatamente refrigerati $\mathrm{a}+4^{\circ} \mathrm{C}$ per massimo 3 giorni e infine trasportati in continuità termica al laboratorio dell'IZS-UM, dove sono stati prontamente congelati a $-18^{\circ} \mathrm{C}$ ed in breve tempo liofilizzati. La stabilità delle specie chimiche durante la movimentazione e lo stoccaggio del campione prima delle analisi è infatti un prerequisito fondamentale per ottenere risultati affidabili, in particolare per le analisi di speciazione, dovendosi evitare, anche in questa fase, che intervengano modifiche nella ripartizione delle varie forme chimiche. Il congelamento in laboratorio dei campioni e la loro liofilizzazione in attesa dell'analisi di speciazione è stata valutata (Dahl et al., 2010) come procedura compatibile con l'esigenza di tale tipo di stabilità. Lo schema di analisi di speciazione utiliz-

Tabella 1. Distribuzione ( \pm deviazione standard; $\mathrm{mg} / \mathrm{kg}$ ) dell'Astot, dell'Asinorg e della sua quantificazione proporzionale sull'Astot in 80 campioni di molluschi, crostacei e pesci della laguna di Boi Cerbus in Sardegna pescati/raccolti nel corso del 2013.

\begin{tabular}{|c|c|c|c|c|}
\hline Denominazione italiana (nome scientifico) & $\mathbf{N}$ & $\mathrm{As}_{\text {tot }}$ & $\mathrm{As}_{\text {inorg }}$ & $A s_{\text {inorg }} / \mathrm{As}_{\text {tot }}$ \\
\hline Cuore (Cerastoderma glaucum) & 11 & $15,47 \pm 2,56$ & $0,111 \pm 0,025$ & $0,74 \pm 0,22$ \\
\hline Granchio da moleca (Carcinus aestuarii) & 10 & $40,04 \pm 12,56$ & $0,054 \pm 0,054$ & $0,14 \pm 0,14$ \\
\hline Murice (Phyllonotus trunculus) & 10 & $105,87 \pm 24,01$ & $0,011 \pm 0,012$ & $0,01 \pm 0,01$ \\
\hline Ghiozzo gò (Zosterisessor ophiocephalus) & 3 & $34,34 \pm 5,71$ & $0,004 \pm 0,003$ & $0,01 \pm 0,01$ \\
\hline Ghiozzo nero (Gobius niger jozo) & 8 & $16,58 \pm 4,92$ & $0,001 \pm 0,001$ & 0,00 \\
\hline Sogliola (Solea vulgaris) & 6 & $15,40 \pm 5,72$ & 0,001 & 0,00 \\
\hline Orata (Sparus aurata) & 3 & $15,20 \pm 1,87$ & 0,001 & 0,00 \\
\hline Anguilla (Anguilla anguilla) & 3 & $6,65 \pm 5,71$ & 0,001 & $0,01 \pm 0,01$ \\
\hline Cefalo o volpina (Mugil cephalus) & 6 & $5,21 \pm 1,16$ & $0,002 \pm 0,002$ & $0,05 \pm 0,04$ \\
\hline Cefalo dorato o lotregano (Liza aurata) & 6 & $3,68 \pm 1,13$ & $0,001 \pm 0,001$ & $0,02 \pm 0,02$ \\
\hline Latterino (Atherina boyeri) & 4 & $2,99 \pm 0,66$ & 0,001 & $0,04 \pm 0,04$ \\
\hline Bavosa basilisco (Salaria basiliscus) & 3 & $2,56 \pm 1,73$ & $0,007 \pm 0,002$ & $0,33 \pm 0,15$ \\
\hline Spigola o branzino (Dicentrarchus labrax) & 5 & $2,00 \pm 1,01$ & 0,000 & $0,01 \pm 0,01$ \\
\hline Salpa (Sarpa salpa) & 2 & $0,49 \pm 0,07$ & 0,000 & 0,00 \\
\hline
\end{tabular}

$\mathrm{As}_{\text {tot }}$, arsenico totale; $\mathrm{As}_{\text {inorg }}$, arsenico inorganico. 
zato ha previsto due procedure autonome: quella per la determinazione della concentrazione di $\mathrm{As}_{\text {tot }}$ (con tecnica ICP-MS) e, in parallelo, quella per la speciazione dell'As (con tecnica combinata HPLC-ICP-MS). Si è quindi proceduto alle analisi con una dotazione strumentale specifica costituita da: una pre-colonna ed una colonna HPLC (ICSep ION-120， 4,6 $\mathrm{mm}$, lunghezza $120 \mathrm{~mm}$ ), uno strumento per HPLC (Perkin Elmer Flexar Liquid Chromatography) ed uno strumento per ICPMS (Perkin Elmer Elan DRC-II). L'accoppiamento online dell'HPLC con l'ICPMS, attraverso connessione del capillare in uscita dalla colonna cromatografica con la camera di nebulizzazione dell'ICP-MS, è stato gestito tramite PC dotato di specifico software (Chromera). Per l'analisi dell'As ${ }_{\text {tot }}$, i sub-campioni sono stati sottoposti a digestione in microonde con $6 \mathrm{~mL}$ di $\mathrm{HNO}_{3}$ concentrato e 2 $\mathrm{mL}$ di $\mathrm{H}_{2} \mathrm{O}_{2}$ al $30 \%$. Per l'estrazione e l'analisi dell'As $_{\text {inorg }}$, i sub-campioni sono stati miscelati con una soluzione $9 / 1$ di $\mathrm{HCl} 0,07 \mathrm{M} \mathrm{e}_{2} \mathrm{O}_{2}$ al $3 \%$ e riscaldati in microonde. Prima della separazione cromatografia gli estratti sono stati centrifugati (circa 4000 rpm per 10 minuti) e filtrati $(\varnothing 0,45 \mu \mathrm{m})$. Tutti i campioni sono stati analizzati in doppio (il valore riferito è quindi quello medio). Relativamente all'analisi statistica dei dati, per i confronti temporali è stato utilizzato il test non parametrico di Kruskal-Wallis (o One-Way ANOVA by Ranks) e per i confronti tra gruppi di organismi marini (per habitat e per taxon) il test, ancora non parametrico, di Mann-Witney Wilcoxon Rank-Sum test).

\section{Risultati}

La tecnica di accoppiamento HPLC-ICP-MS secondo la procedura della quale ci si è avvalsi (Sloth et al., 2005), applicata anche ad organismi marini eduli della laguna di Boi Cerbus, ha confermato che i composti chimici dell'As presenti in concentrazione di gran lunga superiore sono quelli organici. Le concentrazioni delle forme inorganiche sono così basse da non essere rilevabili 0 , se lo sono, rappresentano frazioni percentuali sull'As tot $_{\text {che }}$ arrivano al massimo all'1\%, com'è il caso dei molluschi bivalvi. Come si può rilevare anche dalla Tabella 1, le concentrazioni relativamente

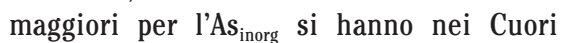
(Cerastoderma glaucum), cui seguono i granchi da moleca (Carcinus aestuarii), mentre tutte le altre specie presentano concentrazioni inferiori e tra loro confrontabili. I risultati del presente studio sono coerenti con quanto riporta la letteratura (Neff, 1997; De Gieter et al., 2002; Sörös et al., 2003; Larsen et al., 2005; Sloth et al., 2005; Greene e Crecelius, 2006) che, come si è più volte rimarcato, riferisce valori assoluti e percentuali molto bassi di $\mathrm{As}_{\text {inorg }}$ per i prodotti della pesca.

\section{Discussione}

Relativamente all'analisi di speciazione dell'As adottata in questo studio, va sottolineato come la fase di estrazione rivesta un'importanza decisiva, considerata la necessità di mantenere la distribuzione originale delle specie chimiche presenti nel campione. Ogni conversione delle specie organiche dell'As, nettamente predominanti nei prodotti della pesca, in specie inorganiche durante la procedura di pretrattamento del campione comprometterebbe infatti la precisione riferita alla proporzione dell'As $_{\text {inorg }}$ sul totale. Tuttavia, relativamente alla specifica procedura utilizzata ed all'obiettivo sotteso, è da ritenersi ammissibile la conversione di composti organici dell'As in altri composti organici del medesimo elemento. La procedura adottata garantisce infatti sul fatto che, dai dati sperimentali disponibili (Sloth $e t$ al., 2005), non sia stata osservata alcuna conversione delle specie madri di As organico in $\mathrm{As}_{\text {inorg, }}$ mentre sono state rilevate possibili inter-conversioni tra alcuni dei composti organici dell'As, precisando che tale eventualità non influenza il contenuto originario di $\mathrm{As}_{\text {inorg. }}$. Inoltre, col termine di arsenico inorganico $\left(\mathrm{As}_{\text {inorg }}\right)$ si intende la somma di As(III) e As(V) poiché, durante il trattamento a microonde, avviene la conversione quantitativa della prima forma nella seconda e la frazione di As(III) dell'acido arsenioso viene tutta ossidata in As(V) dell'acido arsenico. Si consideri a tal proposito che la separazione delle specie inorganiche bersaglio [somma di As(III) + $\mathrm{As}(\mathrm{V})$ ] da quelle organiche rappresenta proprio l'obiettivo di speciazione che ci si è posti, come informazione fondamentalmente utile per le finalità del risk assessment in sanità pubblica. Relativamente all'analisi statistica dei dati, l'utilizzo del test di Kruskal-Wallis, al quale si è ricorsi per la valutazione di eventuali trends stagionali, non ha evidenziato differenze statisticamente significative, né sulle concentrazioni di $\mathrm{As}_{\text {tot }}(\mathrm{P}=0,2549)$, né sulle percentuali medie di $\mathrm{As}_{\text {inorg }}$ sul totale $(\mathrm{P}=0,0817)$, fra le tre calate di pesca/raccolta. Al fine di valutare l'eventuale influenza degli habitat, le 14 specie sono state raggruppate in due cluster: gli organismi bentonici (rappresentati da cuori, murici, granchi, ghiozzi, bavose e sogliole) $v s$ quelli nectonici (con latterini, salpe, cefali, lotregani, orate e spigole). Attraverso l'utilizzo del test non parametrico di Mann-Whitney, la differenza dei livelli medi di $\mathrm{As}_{\text {tot }}$ tra gli organismi bentonici $(38,53 \pm 5,21)$ $v s$ quelli nectonici $(4,89 \pm 0,80)$ è risultata statisticamente significativa $(\mathrm{P}<0,0001)$. Le stesse specie sono state raggruppate in base al taxon in due nuovi cluster: gli invertebrati (cuori, murici e granchi) vs i vertebrati (le restanti specie, rappresentate da pesci Teleostei). Sempre ricorrendo al test non parametrico di Mann-Whitney, anche la differenza dei livelli medi di $\mathrm{As}_{\text {tot }}$ tra gli invertebrati $(52,56 \pm 7,46)$ vs i vertebrati $(9,75 \pm 1,33)$ è risultata statisticamente significativa $(\mathrm{P}<0,0001)$. Con i dati al momento disponibili, non è stato però possibile scomporre il peso attribuibile alla differenza di habitat piuttosto che a quella di taxon ma, plausibilmente, entrambi i fattori sono da ritenersi dei determinanti causali significativi. Relativamente ai livelli medi percentuali di $\mathrm{As}_{\text {inorg }}$ sul totale tra gli organismi bentonici $(0,21 \pm 0,04 \%)$ vs quelli nectonici $(0,02 \pm 0,01 \%)$, sempre ricorrendo al test non parametrico di Mann-Whitney, la differenza non è risultata statisticamente significativa $(\mathrm{P}=0,1038)$, mentre la differenza dei livelli medi percentuali di $\mathrm{As}_{\text {inorg }}$ sul totale tra gli invertebrati $(0,31 \pm 0,06 \%)$ vs i vertebrati $(0,03 \pm 0,01 \%)$ è risultata statisticamente significativa $(\mathrm{P}=0,0002)$. Tale ultimo riscontro, considerata l'ampia premessa svolta sulla chimica e sull'ecologia dell'As negli ambienti marini, può trovare una plausibile ipotesi giustificativa sempre nelle differenze intrinseche, biologiche e filogenetiche, esistenti tra gli invertebrati acquatici ed i pesci, e verosimilmente trovare spiegazione, pur non disponendo col presente studio di evidenze con una sufficiente significatività statistica, anche nelle marcate differenze di habitat tra i due gruppi di organismi indagati (che vede gli invertebrati occupare permanentemente il benthos, mentre i pesci si presentano naturalmente più mobili $e$ tendenti ad occupare, seppur in modo differenziato, tutta la colonna d'acqua, dominio del necton). Si consideri a tale proposito che la maggior attitudine all'accumulo, in termini quantitativi, dell'As $s_{\text {inorg }}$ in ambienti marini da parte degli invertebrati rispetto ai vertebrati è stata descritta ed indagata già da tempo, presupponendo il fatto che l'elemento possa essere accumulato a partire sia dall'acqua, che dal cibo o dai sedimenti, secondo meccanismi differenti a seconda dei taxa. Infatti, non solo le alghe, ma anche i molluschi ed i crostacei (ma scarsamente o per nulla i pesci) sono ritenuti capaci di accumulare arseniato direttamente dall'acqua di mare (Maher e Butler, 1988), secondo modelli di assorbimento proporzionale alla sua concentrazione, almeno fino ad un valore soglia (oltre il quale l'assorbimento di arseniato sarebbe inibito 0 , comunque, indipendente dalla sua concentrazione nell'acqua marina). Quando invece l'elemento si accumula attraverso il cibo, le differenze di concentrazione tra le diverse specie marine (e nelle stesse specie in ambienti diversi) possono essere direttamente correlate alla dieta, tenuto conto che l'assorbimento dell'As tot $_{\text {p }}$ per via alimentare risulta essere la modalità più 
efficiente e che, in tale forma di assunzione, $i$ composti organici dell'elemento vengono assimilati a preferenza dell'As inorg $_{\text {(Maher e }}$ Butler, 1988). Le forme organiche dell'arsenico, come ampiamente è stato riferito nella premessa, si ritiene siano infatti il prodotto della mesofauna associata ad alghe e bioti nei loro habitat naturali (Edmonds e Francesconi, 1987). Un altro aspetto da considerare, anche se il fenomeno del bioaccumulo dell'elemento si verifica a tutti i livelli trofici, è il fatto che non viene rilevata la sua biomagnificazione verso i livelli alti della catena alimentare (Maher e Butler, 1988). A ciò si aggiunga come siano i sedimenti marini a rappresentare il più grande serbatoio di $\mathrm{As}_{\text {inorg }}$ nei sistemi acquatici e che le concentrazioni di tale forma chimica dell'elemento possano diventare elevate in alcuni estuari e aree costiere adiacenti a zone industriali 0 ad aree minerarie e metallurgiche (Maher e Butler, 1988). Nei sedimenti marini l'As si trova infatti soprattutto in forma inorganica, come arsenato 0 arsenito, associato principalmente agli ossidi/idrossidi di ferro e manganese (Sharma e Sohn, 2009) e, anche nei bassi fondali lagunari, i sedimenti non sono stati descritti capaci di svolgere un ruolo primario nel condizionare la qualità dell'acqua sovrastante in relazione ai tenori di $\mathrm{As}_{\text {inorg. }}$. Per contro, le correlazioni delle concentrazioni di $\mathrm{As}_{\text {inorg }}$ nei tessuti di organismi bentonici, bivalvi in particolare, rispetto a quelle dei sedimenti hanno consentito di dimostrare (Maher e Butler, 1988) la capacità di tali organismi acquatici di ingerire una frazione di particolato legata all'As $s_{\text {inorg }}$ nei sedimenti medesimi.

\section{Conclusioni}

I risultati di questo preliminare studio che, ai fini di un corretto risk assessment, rappresentano un utile contributo alla conoscenza dei livelli naturali e della speciazione chimica dell'As negli organismi marini eduli presenti in zone costiere prossime a siti industriali e ad aree minerarie dismesse, nel contempo suggeriscono la necessità di uno sviluppo delle indagini sulla portata del fenomeno e sulle sue implicazioni nelle dinamiche ambientali e sul destino metabolico dell'arsenico inorganico nei diversi organismi marini eduli ivi presenti.

\section{Bibliografia}

Borak J, Hosgood HD, 2007. Seafood arsenic: implications for human risk assessment. Regul Toxicol Pharm 47:204-12.

Dahl L, Molin M, Amlund H, Meltzer HM, Julshamn K, Alexander J, Sloth JJ, 2010.
Stability of arsenic compounds in seafood samples during processing and storage by freezing. Food Chem 123:720-7.

De Gieter M, Leermakers M, Ryssen R, Noyen J, Goeyens L, Baeyens W, 2002. Total and toxic arsenic levels in North Sea fish. Arch Environ Con Tox 43:406-17.

Edmonds JS, Francesconi KA, 1987. Transformations of arsenic in the marine environment. Experentia 43:553-6.

EFSA, 2009. Scientific opinion on arsenic in food (Question No EFSA-Q-2008-425). EFSA J 7/1351:1-199.

EFSA, 2014. Scientific report on dietary exposure to inorganic arsenic in the European population (Question No EFSA-Q-201200107). EFSA J 12/3597:1-68.

Fattorini D, Alonso-Hernandez CM, DiazAsencio M, Munoz-Caravaca A, Pannacciulli FG, Tangherlini M, Regoli F, 2004. Chemical speciation of arsenic in different marine organisms: importance in monitoring studies. Mar Environ Res 58:845-50.

Fattorini D, Notti A, Regoli F, 2006. Characterization of arsenic content in marine organisms from temperate, tropical, and polar environments. Chem Ecol 22:40514.

Foster S, Maher W, Taylor A, Krikowa F, Telford $\mathrm{K}, 2005$. Distribution and speciation of arsenic in temperate marine saltmarsh ecosystems. Environ Chem 2:177-89.

Francesconi KA, 2010. Arsenic species in seafood: origin and human health implications. Pure Appl Chem 82:373-81.

Francesconi KA, Edmonds JS, 1998. Arsenic species in marine samples. Croat Chem Acta 71:343-59.

Francesconi KA, Kuehnelt D, 2004. Determination of arsenic species: a critical review of methods and applications, 20002003. Analyst 129:373-95.

Goessler W, Maher W, Irgolic KJ, Kuehnelt D, Schlagenhaufen C, Kaise T, 1997. Arsenic compounds in a marine food chain. Fresen J Anal Chem 359:434-7.

Gong Z, Lu X, Ma M, Watt C, Le XC, 2002. Arsenic speciation analysis. Talanta 58:7796.

Greene R, Crecelius E, 2006. Total and inorganic arsenic in mid-Atlantic marine fish and shellfish and implications for fish advisories. Integr Environ Assess Manag 2:34454.

Hirata S, Toshimitsu H, Aihara M, 2006. Determination of arsenic species in marine samples by HPLC-ICP-MS. Anal Sci 22:3943.

Kirby J, Maher W, 2002. Tissue accumulation and distribution of arsenic compounds in three marine fish species: relationship to trophic position. Appl Organomet Chem 16:108-15.
Kirby J, Maher W, Spooner D, 2005. Arsenic occurrence and species in near-shore macroalgae-feeding marine animals. Environ Sci Technol 39:5999-6005.

Larsen EH, Sloth JJ, Hansen M, Jorhem L, Engman J, 2005. Determination of inorganic arsenic in white fish using microwaveassisted alkaline alcoholic sample dissolution and HPLC-ICPMS. Anal Bioanal Chem 381:339-46.

Le XC, Cullen WR, Reimer KJ, 1994. Speciation of arsenic compounds in some marine organisms. Environ Sci Technol 28:1598604.

Maher W, Butler E, 1988. Arsenic in the marine environment. Appl Organomet Chem 2:191214.

Moreda-Piñeiro J, Alonso-Rodríguez E, Romarís-Hortas V, Moreda-Piñeiro A, López-Mahia P, Muniategui-Lorenzo S, Prada-Rodríguez D, Bermejo-Barrera P, 2012. Assessment of the bioavailability of toxic and non-toxic arsenic species in seafood samples. Food Chem 130:552-60.

Neff JM, 1997. Ecotoxicology of arsenic in the marine environment. Environ Toxicol Chem 16:917-27.

Piras PL, Mulas GF, 2011. Valutazione dell'esposizione alimentare a contaminanti presenti su bioti eduli (pesci, molluschi bivalvi e crostacei) in un ambiente costiero antistante un'area mineraria ed industriale della Sardegna (Sulcis-Iglesiente). Ital J Food Safety 1:31-6.

Rahman MA, Hasegawa H, Lim RP, 2012. Bioaccumulation, biotransformation and trophic transfer of arsenic in the aquatic food chain. Environ Res 116:118-35.

Saha JC, Dikshit AK, Bandyopadhya M, Saha KC, 1999. A review of arsenic poisoning and its effects on human health. Crit Rev Env Sci Tec 29:281-313.

Sele V, Sloth JJ, Lundebye AC, Larsen EH, Berntssen MHG, Amlund H, 2012. Arsenolipids in marine oils and fats: a review of occurrence, chemistry and future research needs. Food Chem 133:618-30.

Sharma VK, Sohn M, 2009. Aquatic arsenic: toxicity, speciation, transformations, and remediation. Environ Int 35:743-59.

Sloth JJ, Larsen EH, Julshamn K, 2005. Survey of inorganic arsenic in marine animals and marine certified reference materials by anion exchange high-performance liquid chromatography-inductively coupled plasma mass spectrometry. J Agr Food Chem 53:6011-8.

Sörös C, Tünde Bodó E, Fodor P, Morabito R, 2003. The potential of arsenic speciation in molluscs for environmental monitoring. Anal Bioanal Chem 377:25-31. 